\title{
Essential oil composition and antibacterial activities of Gypsophila species
}

\author{
Hüseyin Servi ${ }^{(D)}{ }^{*}$, Betül Eren Keskin ${ }^{(D)}$, Kaan Yılancıoğlu ${ }^{(D)}$, Sezgin Çelik ${ }^{(D)}$
}

\footnotetext{
${ }^{1}$ Altınbas University, Faculty of Pharmacy Pharmaceutical Botany Department, İstanbul, Turkey

${ }^{2}$ Uskudar University, Faculty of Engineering and Natural Sciences Molecular Biology and Genetics Department, İstanbul, Turkey

${ }^{3}$ Uskudar University, Faculty of Engineering and Natural Sciences Chemical and Biological Engineering Department, İstanbul, Turkey

${ }^{4}$ Y1ld1z Technical University, Faculty of Arts \& Science Molecular Biology and Genetic Department, İstanbul, Turkey
}

\begin{abstract}
Essential oil composition of Gypsophila turcica Hamzaoğlu, Gypsophila pinifolia Boiss. \& Hausskn., G. tuberculosa Hub.-Mor., G. eriocalyx Boiss. and G. laricina Schreb. were analyzed by means of gas chromatographymass spectrometry (GC-MS). Thirty six, fourty four, sixty six, forty one and sixty one compounds were identified in the essential oils of G. turcica, G. pinifolia, G. laricina, G. tuberculosa and G. eriocalyx respectively. The major components were determined hentriacontane $(12.93 \pm 0.4 \%)$, 1-octadecanol $(8.97 \pm 0.1 \%)$, hexahydrofarnesyl acetone $(6.9 \pm 0.09 \%)$ and pentacosane $(6.63 \pm 0.08 \%)$ in $G$. turcica oil, hexadecanoic acid $(17.6 \pm 0.4 \%), 1$-tetradecanol $(7.6 \pm 0.1 \%)$ and phytol $(5.63 \pm 0.05 \%)$ in G. pinifolia oil, octacosane $(6.83 \%)$, eicosanal $(6.19 \%)$, triacontane $(6.03 \%)$ and heneicosane $(5.78 \%)$ for G. eriocalyx, hexadecanoic acid $(25.3 \%, 27.0 \%)$ and hentriacontane $(13.0 \%, 12.6 \%)$ for $G$. tuberculosa and $G$. laricina, respectively. Antibacterial activity of G. eriocalyx, G. tuberculosis and G. laricina were investigated against Gram negative (Escherichia coli) and Gram positive (Staphylococcus aureus) bacteria.
\end{abstract}

\section{ARTICLE HISTORY}

Received: September 09, 2018

Revised: November 30, 2018

Accepted: January 09, 2019

\section{KEYWORDS}

Gypsophila,

Essential oil,

Antibacterial activity,

GC-MS

\section{INTRODUCTION}

Gypsophila is the $3^{\text {th }}$ biggest genus in family of Caryophyllaceae to Turkey. Gypsophila species are annual, biennial or perennial herbaceous plants. This genus are distributed mainly in Mediterranean and Iran-Turan areas in Turkey. Gypsophila has 56 species in 10 sections and 33 species are endemic to Turkey [1]. By this way, it has made a significant contribution to the biodiversity of Turkey [2]. Gypsophila turcica is perennial plant and it was described as a new species in 2012 [3].

Gypsophila species are rich source of triterpene saponin especially in root parts $[4,5]$. Triterpene saponin from this genus are used commercially as medicines, detergent, adjuvants and cosmetics [5,6]. Root and barks of the genus used as analgesic, sedative, antipyretic,

\footnotetext{
${ }^{*}$ CONTACT: Hüseyin Servi $\square$ huseyin.servi@altinbas.edu.tr $\equiv$ Altınbas University, Faculty of Pharmacy Pharmaceutical Botany Department, İstanbul, Turkey
} 
antiinflammatory, emetic and insecticidal in Turkey [7]. Biological activities of the genus seem to be associated with triterpene saponin. Due to the various beneficial biological activities, Gypsophila was the focus of studies that described the phytochemistry of the genus extensively.

According to study from Iran, antimicrobial activity and chemical constituents of the essential oils from flower, leaf and stem of Gypsophila bicolor were investigated. The main components of the essential oil from flower were germacrene-D (21.2\%), p-cymene $(20.6 \%)$, bicyclogermacrene $(17.6 \%), \gamma$-dodecadienolactone $(13.7 \%)$ and terpinolene $(9.4 \%)$. The main components of the essential oil from leaves were germacrene-D $(23.4 \%)$, terpinolene $(14.5 \%)$, bicyclogermacrene $(7.5 \%), \gamma$-dodecadienolactone $(6.8 \%), p$-cymene $(6.7 \%)$ and cis- $\beta$ ocimene $(6.3 \%)$. The main components of the essential oil from stems were $\gamma$ dodecadienolactone (28.5\%), bicyclogermacrene (14.8\%), germacrene-D (12.6\%), p-cymene $(12.5 \%)$, terpinolene $(11.6 \%)$ and trans- $\beta$-ocimene $(4.2 \%)$. The essential oils had moderate effect on Gram-positive and Gram negative bacteria, but had significant effect on the fungi [8].

As summarized above Gypsophila species have very high medicinal and commercial importance and also contains interesting natural substances. However, according to our literature survey we have not encountered any reports on the essential oil composition of Gypsophila species from Turkey. Additionaly, there is no report on antibacterial activity of essential oils of G. eriocalyx, G. laricina and G. tuberculosa. This prompted us to investigate the essential oil composition and antibacterial activity of Gypsophila genus. To the best of our knowledge this is the first report on the essential oil composition and antibacterial activity of Gypsophila genus.

\section{MATERIALS AND METHODS}

\subsection{Plant Materials}

Plant materials were collected during the flowering period; G. pinifolia on 17.07.2016 from Aşağı Ulupınar town between Darende and Malatya (1300 m), G. turcica on 17.07.2016 from Jipsli Hills Zara-Baglama village in Sivas (1760 m), G. laricina on 17.07.2017 from Ucpinar, Sarkisla in Sivas (1740-1800 m), G. tuberculosa on 16.07.2015 from Aşağı Ulupınar town between Darende and Malatya $(1480 \mathrm{~m})$ and G. eriocalyx on 20.07.2015 from Jipsli Hills Soğuk Çermik way in Sivas $(1440 \mathrm{~m})$ in Turkey by Çelik and Budak. Voucher specimens have been deposited in the Herbarium of Bozok University (Voucher no: Bozok HB 3310 and Bozok HB 3309 for G. pinifolia and G. turcica respectively), Turkey.

\subsection{Isolation of the Essential Oils}

Aerial parts of the air dried plants subjected to hydrodistillation for $3 \mathrm{~h}$, using a Clevenger-type apparatus to produce essential oils. Condenser of the Clevenger was attached to a microchiller that set to $4^{\circ} \mathrm{C}$. Essential oil yields obtained from G. pinifolia, G. turcica, $G$. laricina, G. eriocalyx and G. tuberculosa 0.03;0.01;0.01;0.01;0.03\% (v/w), respectively. The oils were recovered with $1 \mathrm{~mL} n$-hexane and preserved in amber vials under $-20^{\circ} \mathrm{C}$ until the day they were analyzed.

\subsection{Gas Chromatography/Mass Spectrometry Analysis}

The GC-MS analysis was performed with an Agilent 5975C Inert XL EI/CI MSD system operating in EI mode. Essential oil of G. pinifolia and G. turcica were diluted 1/65 and 1/100 $(\mathrm{v} / \mathrm{v})$ with $n$-hexane, respectively. Injector and MS transfer line temperatures were set at $250^{\circ} \mathrm{C}$. Innowax FSC column (60 m (x) $0.25 \mathrm{~mm}, 0.25 \mu \mathrm{m}$ film thickness) and helium as carrier gas (1 $\mathrm{mL} / \mathrm{min}$ ) were used in both GC/MS analyses. Splitless injection was employed. Oven temperature was programmed to $60^{\circ} \mathrm{C}$ for $10 \mathrm{~min}$. and raised to $220^{\circ} \mathrm{C}$ at rate of $4^{\circ} \mathrm{C} / \mathrm{min}$. Temperature kept constant at $220^{\circ} \mathrm{C}$ for $10 \mathrm{~min}$. and then raised to $240^{\circ} \mathrm{C}$ at a rate of $1^{\circ} \mathrm{C} / \mathrm{min}$. Mass spectra were recorded at $70 \mathrm{eV}$ with the mass range $\mathrm{m} / \mathrm{z} 35$ to 425 . 


\subsection{Gas Chromatography Analysis}

The GC analyses were done with an Agilent 6890N GC system. FID detector temperature was set to $300^{\circ} \mathrm{C}$ and same operational conditions applied to a duplicate of the same column used in GC-MS analyses. Simultaneous auto injection was done to obtain the same retention times. Relative percentage amounts of the separated compounds were calculated from integration of the peaks in FID chromatograms. Identification of essential oil components were carried out by comparison of their relative retention indices (RRI) obtained by series of $n$ alkanes (C5 to $\mathrm{C} 30$ ) to the literature and with mass spectra comparison [11-27]. Mass spectra comparison was done by computer matching with commercial Wiley 8th Ed./NIST 05 Mass Spectra library, Adams Essential Oil Mass Spectral Library and Pallisade 600K Complete Mass Spectra Library. The analysis was carried out in triplicate and the results were given as the mean \pm standard deviation.

\subsection{Antibacterial Assay}

Antibacterial activities of the essential oils were tested against two strains; Gram positive Staphylococcus aureus (ATCC 25923) and Gram negative Escherichia coli (ATCC 25922). For the antimicrobial tests, Luria-Bertani broth was used as a growth medium for bacteria.

In order to evaluate antibacterial activity, minimum inhibition concentration $\left(\mathrm{MIC}_{50}\right)$ values were determined by using broth dilution method. DMSO was used in stock solutions to enhance solubility of the essential oils. Serial dilutions of the stock solutions were prepared on a 96 well plate. After incubation at $37^{\circ} \mathrm{C}$ for $24 \mathrm{~h}$, bacterial suspension concentrations were standardized to McFarland No: 0.5. Essential oils and bacterial cultures were mixed in the range of $1000-1,95 \mu \mathrm{g} / \mathrm{mL}$ as final concentration. It was paid attention to not exceed $1 \%$ final concentration for DMSO. After treatment, the bacteria were incubated at $37^{\circ} \mathrm{C}$ for $24 \mathrm{~h}$. As negative control, essential oil-free solutions were used. Each test was repeated for three times. Growth analysis was done by using spectrophotometric measurements for MIC determination. Minimum inhibitory concentrations $\left(\mathrm{MIC}_{50}\right)$ were detected as the minimum concentration at which at least $50 \%$ of bacterial growth was missing.

\section{RESULTS}

Essential oil composition of Gypsophila turcica, G. pinifolia, G. tuberculosa, $G$. eriocalyx and $G$. laricina. were analyzed by means of gas chromatography-mass spectrometry (GC-MS). In order, thirty six, fourty four, sixty six, forty one and sixty one compounds were identified in the essential oils of G. turcica, G. pinifolia, G. laricina, G. tuberculosa and $G$. eriocalyx that represent $69.1 \%, 71.7 \%, 78.1 \%, 71.7 \%$ and $85.6 \%$ of the oil, respectively. The major components were determined hentriacontane (12.93\%), 1-octadecanol (8.97\%), hexahydrofarnesyl acetone $(6.9 \%)$ and pentacosane $(6.63 \%)$ in G. turcica oil, hexadecanoic acid (17.6\%), 1-tetradecanol (7.6\%) and phytol (5.63\%) in G. pinifolia oil, octacosane (6.83\%), eicosanal $(6.19 \%)$, triacontane $(6.03 \%)$ and heneicosane $(5.78 \%)$ for $G$. eriocalyx, hexadecanoic acid $(25.3 \%, 27.0 \%)$ and hentriacontane $(13.0 \%, 12.6 \%)$ for $G$. tuberculosa and G. laricina, respectively. The essential oil composition of five Gypsophila species are given in Table 1.

Antibacterial of the oils were evaluated for one Gram (+) and one Gram (-) bacteria by using a broth microdilution assay. G. eriocalyx essential oil showed mild activity on $S$. aureus $(250 \mu \mathrm{g} / \mathrm{mL})$ but the oil showed very low activity against $E$. coli $(1000 \mu \mathrm{g} / \mathrm{mL})$. However, $G$. tuberculosa and $G$. laricina essential oils did not show any significant activity against tested grains. The results of antibacterial activity of Gypsophila species are given in Table 2.

The essential oil of G. pinifolia, G. tuberculosa and G. laricina had hexadecanoic acid in high amount unlike G. turcica and G. eriocalyx. Essential oils of G. turcica, G. tuberculosa and 
$G$. laricina were rich in hentriacontane. But hentriacontane contained at low amount in $G$. eriocalyx and not detected in G. pinifolia.

Table 1. The essential oil composition of five Gypsophila species

\begin{tabular}{|c|c|c|c|c|c|c|c|c|c|}
\hline & & & & aricina & G.tuberculosa & G. turcica & G.eriocalyx & G.pinifolia & \\
\hline No & $\mathbf{R R I}^{1}$ & $\begin{array}{l}\text { RRI } \\
\text { Lit. }^{2}\end{array}$ & Compound & $\begin{array}{l}\text { Mean } \\
(\%)^{3}\end{array}$ & $\begin{array}{l}\text { Mean } \\
(\%)\end{array}$ & $\begin{array}{l}\text { Mean } \\
(\%)\end{array}$ & $\begin{array}{l}\text { Mean } \\
(\%)\end{array}$ & $\begin{array}{l}\text { Mean } \\
(\%)\end{array}$ & Id. Met. $^{4}$ \\
\hline 1 & 1200 & 1200 & Dodecane & - & - & - & - & 0.6 & RI, MS, Ac \\
\hline 2 & 1233 & 1244 & 2-pentyl furan & 0.27 & - & - & 0.08 & - & RI, MS \\
\hline 3 & 1300 & 1300 & Tridecane & - & - & - & 0.09 & - & RI, MS, Ac \\
\hline 4 & 1398 & 1399 & Nonanal & 0.29 & 0.33 & - & 0.32 & 0.33 & RI, MS \\
\hline 5 & 1401 & 1400 & Tetradecane & 0.16 & 0.26 & 0.4 & 0.35 & 0.8 & RI, MS, Ac \\
\hline 6 & 1442 & 1443 & Dimethyl tetradecane & 0.06 & - & - & - & - & RI, MS \\
\hline 7 & 1498 & 1466 & $\alpha$-cubebene & - & - & - & - & 0.1 & RI, MS \\
\hline 8 & 1499 & 1505 & Dihydroedulan II & 0.15 & - & - & - & - & RI, MS \\
\hline 9 & 1502 & 1500 & Pentadecane & 0.15 & 0.26 & 0.6 & 0.1 & 0.2 & RI, MS, Ac \\
\hline 10 & 1505 & 1506 & Decanal & 0.47 & 1.05 & 0.5 & 1.79 & 2.53 & RI, MS \\
\hline 11 & 1510 & 1516 & Theaspirane A & 0.70 & - & 0.5 & 0.04 & 0.1 & RI, MS \\
\hline 12 & 1525 & 1532 & Camphor & 0.04 & - & - & - & - & RI, MS \\
\hline 13 & 1529 & 1535 & Dihydroedulan I & 0.14 & - & - & - & - & RI, MS \\
\hline 14 & 1536 & 1541 & Benzaldehyde & - & - & - & 0.07 & - & RI, MS \\
\hline 15 & 1543 & 1548 & (E)-2-nonenal & 0.12 & - & - & - & - & RI, MS \\
\hline 16 & 1549 & 1553 & Theaspirane B & 0.64 & - & 0.4 & 0.18 & - & RI, MS \\
\hline 17 & 1550 & 1553 & $\beta$-Linalool & - & 0.34 & - & - & 2.03 & RI, MS \\
\hline 18 & 1558 & 1549 & 1-Tetradecene & 0.08 & - & - & 0.07 & - & RI, MS \\
\hline 19 & 1580 & 1562 & Longifolene & - & - & - & 0.09 & - & RI, MS \\
\hline 20 & 1602 & 1600 & Hexadecane & 0.29 & 0.34 & 0.77 & 0.45 & 0.80 & RI, MS, Ac \\
\hline 21 & 1603 & 1605 & 2-undecanone & - & - & 0.03 & 0.06 & - & RI, MS \\
\hline 22 & 1608 & 1612 & $\beta$-caryophyllene & - & - & - & 0.13 & 0.1 & RI, MS \\
\hline 23 & 1612 & 1617 & Undecanal & - & - & - & - & 2.57 & RI, MS \\
\hline 24 & 1612 & 1613 & $\beta$-cedrene & - & - & - & 0.13 & - & RI, MS \\
\hline 25 & 1633 & 1638 & $\beta$-cyclocitral & 0.13 & - & - & 0.06 & 0.17 & RI, MS \\
\hline 26 & 1635 & 1644 & Thujopsene & 0.04 & - & - & 0.1 & - & RI, MS \\
\hline 27 & 1649 & 1654 & 1-Hexadecene & - & - & 0.3 & - & - & RI, MS \\
\hline 28 & 1653 & 1655 & (E)-2-Decanal & 0.25 & - & - & 0.45 & 0.3 & RI, MS \\
\hline 29 & 1660 & 1664 & Nonanol & 0.1 & - & - & - & - & RI, MS \\
\hline 30 & 1672 & 1671 & (E)- $\beta$-Farnesene & - & - & - & 0.13 & - & RI, MS \\
\hline 31 & 1683 & 1687 & a-Humulene & - & - & - & 0.07 & - & RI, MS \\
\hline 32 & 1693 & 1685 & $\begin{array}{l}\text { 6,10-dimethyl-2- } \\
\text { undecanone }\end{array}$ & 0.1 & - & - & - & - & RI, MS \\
\hline 33 & 1701 & 1700 & Heptadecane & 0.28 & 0.33 & 0.50 & 0.39 & - & RI, MS, Ac \\
\hline 34 & 1703 & 1706 & $\alpha$-terpineol & - & - & - & - & 0.80 & RI, MS \\
\hline 35 & 1718 & 1722 & Dodecanal & 0.29 & 0.28 & - & 0.67 & 0.53 & RI, MS \\
\hline 36 & 1735 & 1742 & $\beta$-Selinene & - & 0.23 & - & - & - & RI, MS \\
\hline 37 & 1761 & 1763 & Naphthalene & 0.32 & - & - & - & - & RI, MS \\
\hline 38 & 1764 & 1766 & Decanol & - & - & - & 0.23 & 0.23 & RI, MS \\
\hline 39 & 1775 & 1779 & $(E, Z)$-2,4-Decadienal & 0.13 & 0.13 & - & 0.11 & - & RI, MS \\
\hline
\end{tabular}


Table 1. Continues

\begin{tabular}{|c|c|c|c|c|c|c|c|c|c|}
\hline 40 & 1785 & 1786 & Ar-curcumene & - & - & - & 0.03 & - & RI, MS \\
\hline 41 & 1802 & 1800 & Octadecane & 0.21 & 0.29 & 0.3 & 0.51 & 0.3 & RI, MS, Ac \\
\hline 42 & 1804 & 1820 & Isogeraniol & - & - & - & - & 0.3 & RI, MS \\
\hline 43 & 1815 & 1815 & 2-tridecanone & - & - & 0.1 & - & - & RI, MS \\
\hline 44 & 1823 & 1823 & $(E)$ - $\alpha$-Damascenone & 0.2 & - & - & - & 0.6 & RI, MS \\
\hline 45 & 1824 & 1827 & $(E, E)$-2,4-Decadienal & 0.4 & 0.56 & - & 0.13 & - & RI, MS \\
\hline 46 & 1826 & 1830 & Tridecanal & - & - & - & 0.51 & 2.3 & RI, MS \\
\hline 47 & 1830 & 1838 & $(E)-\beta$-Damascenone & 0.36 & 0.18 & - & - & 1.63 & RI, MS \\
\hline 48 & 1850 & 1857 & Geraniol & - & - & - & - & 1.23 & RI, MS \\
\hline 49 & 1863 & 1868 & $(E)$-Geranyl acetone & 1.12 & 1.17 & 1.03 & 0.6 & 1.43 & RI, MS \\
\hline 50 & 1879 & 1871 & Undecanol & 0.17 & - & - & - & - & RI, MS \\
\hline 51 & 1886 & 1864 & p-cymene-8-ol & 0.08 & - & - & - & - & RI, MS \\
\hline 52 & 1901 & 1900 & Nonadecane & - & 0.73 & - & 1.3 & - & RI, MS, Ac \\
\hline 53 & 1931 & 1933 & Tetradecanal & 0.38 & - & - & 0.96 & 0.5 & RI, MS \\
\hline 54 & 1953 & 1958 & (E)- $\beta$-Ionone & 1.03 & 0.52 & 0.5 & 0.73 & 0.6 & RI, MS \\
\hline 55 & 1969 & 1973 & 1-Dodecanol & 0.63 & 0.88 & - & - & 0.6 & RI, MS \\
\hline 56 & 2003 & 2000 & Eicosane & 0.29 & 0.74 & 0.3 & 1.13 & 0.23 & RI, MS, Ac \\
\hline 57 & 2005 & 2007 & Caryophyllene oxide & 0.29 & - & - & - & - & RI, MS \\
\hline 58 & 2028 & 2036 & 2-pentadecanone & - & 0.41 & 0.3 & - & - & RI, MS \\
\hline 59 & 2037 & 2036 & Pentadecanal & 0.26 & - & - & - & - & RI, MS \\
\hline 60 & 2039 & 2036 & Hexadecanal & - & - & - & - & 2.00 & RI, MS \\
\hline 61 & 2043 & 2050 & (E)-Nerolidol & 0.05 & - & - & - & - & RI, MS \\
\hline 62 & 2051 & 2056 & 13-Tetradecanolide & 0.35 & - & - & - & - & RI, MS \\
\hline 63 & 2104 & 2100 & Heneicosane & - & 0.55 & 0.5 & 5.78 & 0.27 & RI, MS, Ac \\
\hline 64 & 2135 & 2131 & $\begin{array}{l}\text { Hexahydro farnesyl } \\
\text { acetone }\end{array}$ & 1.65 & 1.9 & 6.9 & 4.44 & 2.73 & RI, MS \\
\hline 65 & 2145 & 2136 & Hexadecanal & 0.3 & - & - & 1.01 & - & RI, MS \\
\hline 66 & 2145 & 2148 & $\begin{array}{l}\text { (Z)-3-hexeneyl } \\
\text { benzoate }\end{array}$ & - & - & - & - & 1.36 & RI, MS \\
\hline 67 & 2170 & 2192 & Nonanoic acid & 0.5 & - & - & - & - & RI, MS \\
\hline 68 & 2173 & 2179 & Tetradecanol & - & - & 0.7 & 0.68 & 7.6 & RI, MS \\
\hline 69 & 2184 & 2186 & Eugenol & - & - & - & - & 0.1 & RI, MS \\
\hline 70 & 2190 & 2144 & Spathulenol & 0.05 & - & - & - & 1.1 & RI, MS \\
\hline 71 & 2190 & 2198 & 1-Docosene & - & 2.21 & - & - & - & RI, MS \\
\hline 72 & 2202 & 2200 & Docosane & - & 0.6 & 0.4 & 1.6 & - & RI, MS, Ac \\
\hline 73 & 2225 & 2226 & $\begin{array}{l}\text { Hexadecanoic acid } \\
\text { methyl ester }\end{array}$ & - & - & - & - & 0.2 & RI, MS \\
\hline 74 & 2240 & 2242 & 2-Heptadecanone & - & 0.19 & - & 0.12 & - & RI, MS \\
\hline 75 & 2278 & 2282 & Decanoic acid & 1.03 & 1.56 & - & - & 1.37 & RI, MS \\
\hline 76 & 2290 & 2296 & Isophytol & - & - & 0.4 & - & - & RI, MS \\
\hline 77 & 2302 & 2300 & Tricosane & 0.55 & 0.81 & 2.2 & 4.5 & - & RI, MS, Ac \\
\hline 78 & 2318 & 2315 & $\begin{array}{l}\text { 2,4-bis-tert- } \\
\text { butylphenol }\end{array}$ & 0.35 & - & 2.23 & - & - & RI, MS \\
\hline 79 & 2338 & 2345 & Galaxolide I & - & - & - & 0.13 & - & RI, MS \\
\hline 80 & 2345 & 2353 & Galaxolide II & - & - & - & 0.09 & - & RI, MS \\
\hline 81 & 2355 & 2353 & Octadecanal & 0.28 & - & 0.9 & 1.71 & - & RI, MS \\
\hline 82 & 2381 & 2384 & 1-Hexadecanol & - & - & - & 0.4 & - & RI, MS \\
\hline
\end{tabular}


Table 1. Continues

\begin{tabular}{|c|c|c|c|c|c|c|c|c|c|}
\hline 83 & 2384 & 2381 & Farnesyl acetone & 1.41 & 1.5 & 1.87 & 0.8 & 2.1 & RI, MS \\
\hline 84 & 2402 & 2400 & Tetracosane & 0.31 & 0.63 & 0.6 & 0.5 & - & RI, MS, Ac \\
\hline 85 & 2448 & 2471 & Nonadecanal & 0.2 & - & - & - & - & RI, MS \\
\hline 86 & 2489 & 2492 & Dodecanoic acid & 3.51 & 7.55 & - & 0.17 & 1.9 & RI, MS \\
\hline 87 & 2504 & 2500 & Pentacosane & 1.4 & 3.06 & 6.63 & 2.32 & - & RI, MS, Ac \\
\hline 88 & 2555 & 2592 & Diisobutyl phthalate & 2.15 & 4.23 & 4.48 & 1.42 & 2.91 & RI, MS \\
\hline 89 & 2585 & 2582 & Eicosanal & 2.07 & - & - & 6.19 & - & RI, MS \\
\hline 90 & 2589 & 2607 & 1-octadecanol & - & 0.88 & 8.97 & 0.63 & - & RI, MS \\
\hline 91 & 2590 & 2617 & Tridecanoic acid & 0.23 & 0.37 & - & - & - & RI, MS \\
\hline 92 & 2606 & 2600 & Hexacosane & 0.31 & 0.58 & - & 0.32 & - & RI, MS, Ac \\
\hline 93 & 2618 & 2622 & Phytol & 1.76 & 1.1 & 2.7 & 2.59 & 5.63 & RI, MS \\
\hline 94 & 2671 & 2676 & Heneicosanal & 1.97 & - & - & - & - & RI, MS \\
\hline 95 & 2701 & 2704 & Tetradecanoic acid & 4.7 & 6.53 & - & 0.26 & 1.33 & RI, MS \\
\hline 96 & 2706 & 2700 & Heptacosane & 0.7 & 1.97 & 1.3 & 3.40 & 1.27 & RI, MS, Ac \\
\hline 97 & 2775 & 2783 & 1-Docosanol & 0.31 & - & 0.8 & - & - & RI, MS \\
\hline 98 & 2796 & 2794 & 1-Eicosanol & - & - & 3.63 & - & - & RI, MS \\
\hline 99 & 2795 & 2800 & Octacosane & 0.25 & - & - & 6.83 & - & RI, MS \\
\hline 100 & 2806 & 2822 & Pentadecanoic acid & 1.4 & 1.69 & - & - & 0.60 & RI, MS \\
\hline 101 & 2838 & 2857 & Palmito-y-lactone & 0.21 & 0.41 & - & 0.25 & - & RI, MS \\
\hline 102 & 2904 & 2900 & Nonacosane & - & - & 1.37 & 1.65 & - & RI, MS, Ac \\
\hline 103 & 2918 & 2931 & Hexadecanoic acid & 27.03 & 25.3 & - & 4.64 & 17.6 & RI, MS \\
\hline 104 & 2984 & 2990 & Docosanal & 0.22 & - & 0.73 & - & - & RI, MS \\
\hline 105 & 3003 & 3000 & Triacontane & - & - & 2.4 & 6.03 & - & RI, MS, Ac \\
\hline 106 & 3098 & 3100 & Hentriacontane & 12.63 & 13.0 & 12.93 & 1.20 & - & RI, MS, Ac \\
\hline & & & Total & 78.1 & 85.6 & 69.1 & 71.7 & 71.7 & \\
\hline
\end{tabular}

${ }^{1}$ RRI: Relative retention time indices calculated against $n$-alkanes (C5-C30).

${ }^{2}$ RRI Lit.: Relative retention time given in the literature for the compound in similar columns and analysis conditions.

${ }^{3}$ The results of the analysis.

${ }^{4}$ Identification method: RI: identification based on the relative retention times (RRI) of genuine compounds on the HP Innowax column and the literature data; MS: identification based on MS comparison with the database or the literature data, Ac: Identification is done according to RRI and MS values of the authentic compounds.

Table 2. Antibacterial activity [MIC $\left.\mathrm{MI}_{50}(\mu \mathrm{g} / \mathrm{mL})\right]$ of the essential oils of G. eriocalyx, G. tuberculosa and G. laricina

\begin{tabular}{cccc}
\hline Strain & G. eriocalyx $(\mu \mathrm{g} / \mathrm{mL})$ & G. tuberculosa $(\mu \mathrm{g} / \mathrm{mL})$ & G. laricina $(\mu \mathrm{g} / \mathrm{mL})$ \\
\hline E. coli & 1000 & $>1000$ & $>1000$ \\
\hline S. aureus & 250 & $>1000$ & $>1000$ \\
\hline
\end{tabular}

\section{DISCUSSION and CONCLUSION}

Only mild antibacterial activity is observed on G. eriocalyx essential oil against $S$. aureus. The main compounds of essential oil of $G$. eriocalyx contained low amount or not detected in other Gypsophila species. Eicosanal is one of the main compound of G. eriocalyx. Antibacterial activity could be correlated with this compound. According to a study from Iran, Gypsophila bicolor was reported to contain germacrene-D, $p$-cymene, bicyclogermacrene, $\gamma$ dodecadienolactone, terpinolene, cis- $\beta$-ocimene and trans- $\beta$-ocimene [8] however these compounds were not detected in the G. turcica, G. pinifolia, G. eriocalyx, G. tuberculosa and 
G. laricina. These differences in the previous literature and present data could be related to different collection times, climatic and soil conditions, ecological factors, methods and instruments employed in analysis or different genotypes. There are very few reports on the essential oil of Gypsophila species therefore it is difficult to produce a comment on the chemosystematic position of this species according to current findings and the existing reports. We believe the results obtained from this research will stimulate further research on the chemistry of Gypsophila species.

\section{Orcid}

Hüseyin Servi (D) https://orcid.org/0000-0002-4683-855X

Betül Eren Keskin (D) https://orcid.org/0000-0003-2439-2558

Kaan Y1lancioğlu (D) https://orcid.org/0000-0001-7105-0898

Sezgin Çelik (iD) https://orcid.org/0000-0001-7467-7717

\section{REFERENCES}

[1] Davis, P.H., Mill, R.R, Tan, K. \& Edmondson. J.R. (1982). Flora of Turkey and the East Aegean Islands. Edinburgh, Scotland: University Press.

[2] Chandra, S., \& Rawat, D.S. (2015). Medicinal plants of the family Caryophyllaceae: a review of ethno-medicinal uses and pharmacological properties. Integr. Med. Res., 4, 123131.

[3] Hamzaoglu, E. (2012). A new species of Gypsophila and a new name for Silene (Caryophyllaceae) from Turkey. Turk. J. Biol., 36, 135-139.

[4] Henry, M. (1993). Gypsophila paniculata L. (Baby's Breath): in vitro culture and the production of gypsogenin saponins. In: Bajaj YPS, editor. Biotechnology in agriculture and forestry, Berlin/Heidelberg: Springer-Verlag, 21, 187-206.

[5] Hostettmann, K., Marston, A. (1995). Chemistry and Pharmacology of Natural Products, Saponin. Cambridge, UK: Cambridge University Press.

[6] Gevrenova, R., Stancheva, T., Voynikov, Y., Laurain-Mattar, D., \& Henry, M. (2010). Root in vitro cultures of six Gypsophila species and their saponin contents. Enzyme. Microb. Tech., 47, 97-104.

[7] Yücekutlu, A.N., \& Bildac1, I. (2008). Determination of Plant Saponins and Some of Gypsophila Species: A review of the literature. Hacettepe J Biol Chem., 36, 129-135.

[8] Shafagha, A. \& Shafaghatlonbar, M. (2011). Antimicrobial activity and chemical constituents of the essential oils from flower, leaf and stem of Gypsophila bicolor from Iran. Nat Prod Commun., 6, 275-276.

[9] Servi, H., Keskin, B.E., Çelik, S., Budak, Ü., Kırmızıtaş, F.C., Bektaş, E.B. (2017). Essential oil composition and fatty acid profile of two endemic Gypsophila species from Turkey. Am J Essent Oil Nat Prod., 5, 16-20.

[10] Servi, H., Keskin, B.E., Çelik, S., Budak, Ü., Kababıyık, B. (2018). Essential Oil and Fatty Acid Composition of Endemic Gypsophila laricina Schreb. from Turkey. Turk J Pharm Sci., In press.

[11] Baser, K.H.C., Demirci, B., Özek, T., Akalin, E., Özhatay, N. (2002). Micro-distilled volatile compounds from Ferulago species growing in western Turkey. Pharm Biol., 40, 466-471.

[12] Demirci, B., Başer, K.H., Yıldız, B., Bahçecioğlu, Z. (2003). Composition of the essential oils of six endemic Salvia spp. from Turkey. Flavour Frag J., 18, 116-121. 
[13] Karamenderes, C., Demirci, B., Başer, K.H. (2008). Composition of Essential Oils of Ten Centaurea L. Taxa from Turkey. J Essent Oil Res., 20, 342-349.

[14] Moronkola, D.O., Ogunwande, I.A., Başer, K.H.C., Ozek, T., Ozek, G. (2009). Essential Oil Composition of Gmelina arborea Roxb., Verbenaceae from Nigeria. J Essent Oil Res., 21, 264-266.

[15] Norouzi-Arasi, H., Yavari, I., Chalabian, F., Kiarostami, V., Ghaffarzadeh, F., Nasirian, A. (2006). Chemical constituents and antimicrobial activities of the essential oil of Acroptilon repens (L.) DC. Flavour Frag J., 21, 247-249.

[16] Polatoğlu, K., Demirci, B., Gören, N., Başer, K.H.C. (2011). Essential oil composition of Tanacetum kotschyi from Turkey. Chem Nat Comp., 47, 297-299.

[17] Polatoğlu, K., Gören, N., Karakoç, Ö.C. (2013). Phytotoxic, DPPH scavenging, insecticidal activities and essential oil composition of Achillea vermicularis, A. teretifolia and proposed chemotypes of A. biebersteinii (Asteraceae). Ind Crop Prod., 51, 35-45.

[18] Polatoğlu, K., Şen, A., Bulut, G., Bitiş, L., Gören, N. (2014). Essential Oil Composition of Centaurea stenolepis Kerner. from Turkey. J Essent Oil Bear Pl., 17, 1268-1278.

[19] Polatoğlu, K., Arsal, S., Demirci, B., Başer, K.H. (2015). DPPH Scavenging, PRAP Activities and Essential Oil Composition of Edible Lathyrus ochrus L. (Cyprus Vetch, Luvana) from Cyprus. J Oleo Sci., 64, 309-314.

[20] Polatoğlu, K., Karakoç, Ö.C., Yücel, Y.Y., Demirci, B., Gören, N., Başer, K.H. (2015). Composition, insecticidal activity and other biological activities of Tanacetum abrotanifolium Druce. essential oil. Ind Crop Prod., 71, 7-14.

[21] Sura, B.E., Demirci, B., Demir, S., Karaalp, C., Başer, K.H. (2013). Composition of the essential oils of Centaurea aphrodisea, C. polyclada, C. athoa, C. hyalolepis and $C$. iberica. J Essent Oil Res., 25, 79-84.

[22] Tabanca, N., Demirci, B., Ozek, T., Kirimer, N., Baser, K.H.C., Bedir, E., Khan, I.A., Wedge, D.E. (2006). Gas chromatographic-mass spectrometric analysis of essential oils from Pimpinella species gathered from Central and Northern Turkey. J Chromatogr A., 1117, 194-205.

[23] Tabanca, N., Demirci, B., Baser, K.H.C., Aytac, Z., Ekici, M., Khan, S.I., Jacob, M.R., Wedge, D.E. (2006). Chemical composition and antifungal activity of Salvia macrochlamys and Salvia recognita essential oils. J Agr Food Chem., 54, 6593-6597.

[24] Viegas, M.C., Bassoli, D.G. (2007). Utilização do índice de retenção linear para caracterização de compostos voláteis em café solúvel utilizando GC-MS e coluna HPInnowax. Quim Nova, 30, 2031-2034.

[25] Tastan, P., Fafal, T., Tüzün, B. S., Gönenç, T., Demirci, B. \& Kivcak, B. (2017). Composition of essential oil and fatty acids of Centaurea pichleri ssp. pichleri. International Journal of Secondary Metabolite, 4, 37-42.

[26] Tuzun, B. S., Gonenc, T., Tastan, P., Fafal, T., \& Demirci, B. (2017). Essential Oil and Fatty Acid Composition of Centaurea solstitialis ssp. solstitialis. International Journal of Secondary Metabolite, 4, 58-62.

[27] Salleh, W.M.N.H.W., Ahmad, F., Yen, K. H. \& Sirat, H.M. (2011). Chemical compositions, antioxidant and antimicrobial activities of essential oils of Piper caninum Blume. International Journal of Molecular Sciences, 12, 7720-7731. 